\title{
A Computational Approach to Predict the Regulation of Antioxidant Enzyme, Catalase in the Plant Defence Mechanism
}

\author{
Michael I. Jesse \\ Department of \\ Biotechnology, \\ University of Madras, \\ Guindy, Chennai - 600025 . \\ India
}

\author{
Dharanivasan. G \\ Department of Biotechnology, \\ University of Madras, \\ Guindy, Chennai - 600025. India
}

\author{
Mohammed Riyaz. \\ S.U \\ Department of \\ Biotechnology, \\ University of Madras, \\ Guindy, Chennai - 600025. \\ India
}

\author{
Rajamuthuramalingam \\ Department of \\ Biotechnology, \\ University of Madras, \\ Guindy, Chennai - 600025 . \\ India
}

\begin{abstract}
Plants defense system protects themselves from pathogens in two ways by preformed mechanisms and through infection-induced responses of the immune system. It is always apparent as a restriction of pathogen growth and spread to a little zone around the infected area. During localized cell death, the visible necrotic lesion evokes up and induces the programmed cell death (apoptosis). Systemic acquired resistance (SAR) will be helpful to the infected plants after a period of 5-7 days. Salicylic acid (SA) is a plant hormone essential for the immunity in plants. SA has been found to involve in the control of microbe/pathogenassociated molecular pattern triggered immunity, effectortriggered immunity and system acquired resistance (SAR). The binding modes and the bonding pattern between salicylic acid and the enzyme catalase is still unknown. In our study, the different binding modes of salicylic acid in different pockets were analyzed. Among the chosen pockets, the best probable binding pocket was identified computationally based on the binding energy, intra-molecular energy, internal energy and inhibition constant between two molecules.
\end{abstract}

\section{Keywords}

Systemic acquired resistance, Binding pocket, Binding energy, Docking

\section{INTRODUCTION}

Immune system is most vital machinery in plants. Plants are sessile and they have developed a wide range of defense or stress responses to protect themselves against biotic and abiotic stresses. The disease resistance in plants is always apparent as a restriction of pathogen growth and spread to a little zone around the infected area. This kind of restriction is accompanied by the localized cell death of host tissue. In various incompatible infections, these reactions are often associated with the death of small number of cells (literally means 'apoptosis') at the site of infection known as 'hypersensitive response' (HR). Followed by the lesion formation, the plants adapt resistance to a vast range of pathogens that encounter the area of infection site. Systemic acquired resistance (SAR) will be helpful to the infected plants after a period of 5-7 days. [1, 2, 3]. SAR is linked typically with the set of pathogenesis-related (PR) proteins. Hence there will be a greater expression of genes that are responsible for the defence mechanism in resistant tissue. PR proteins which are expressed in such a way serves as a marker for disease resistance [4].

\author{
Kathiravan. K. \\ Department of Biotechnology, \\ University of Madras, \\ Guindy, Chennai - 600025. India.
}

Salicylic acid (SA) is a plant hormone essential for the immunity, in plants. SA has been found to involve in the control of (1) microbe/pathogen-associated molecular pattern (MAMP/PAMP)triggered immunity (MTI/PTI), (2) effector-triggered immunity (ETI) and (3) system acquired resistance (SAR). Based on the host-pathogen relationship, SA can promote or repress the hypersensitive response (HR), otherwise called programmed cell death (apoptosis in mammals) [5, 6]. In Arabidopsis thaliana, SA can promote hypersensitivity response mediated programmed cell death after infection with the avirulent Pseudomonas syringae pv. tomato (Pst) avrRpt2, or indirectly through non-expressor of pathogenesis-related (PR) genes 1 (NPR1)-repress cell death after infection with the avirulent Pst DC3000 avrRpml [6, 7]. In addition, salicylic acid can control the oxidative stress by promoting the production of $\mathrm{H}_{2} \mathrm{O}_{2}$ preventing the split into $\mathrm{H}_{2}$ and $\mathrm{O}_{2}$ and thus balance reactive oxygen species (ROS) production inside the chloroplast to particularly disservice programmed cell death [8]. $\mathrm{H}_{2} \mathrm{O}_{2}$ is a reactive oxygen species that are normally produced in chloroplasts and mitochondria as a byproduct of photosynthesis and oxidative phosphorylation, respectively. Under normal growth conditions, chloroplasts are the main source of ROS in photosynthetic tissues, whereas that role is fulfilled by mitochondria in non-photosynthetic tissues [9]. At the instance of biotic stress, SA has been known to inhibit certain enzymes including catalase and ascorbate peroxidase. These enzymes are salicylic acid binding proteins (SABPs) and antioxidant enzymes in tobacco as well to facilitate $\mathrm{H}_{2} \mathrm{O}_{2}$ accumulation. [10, 11, 12].

One of the models confers that salicylic acid (SA) binds to and inactivates catalase, increasing the level of $\mathrm{H}_{2} \mathrm{O}_{2}$ and other ROS and boosts up SAR. This in turn, induces the expression of PR genes - a marker of SAR and inhibits the activity of catalase. In addition, $\mathrm{H}_{2} \mathrm{O}_{2}$ also stimulates $\mathrm{SA}$ production in tobacco plants. In some plants, catalase gene expression has been shown to respond to SA. It has been observed that inhibition of catalase in leaf extracts requires concentrations of SA far above those in uninfected tissues $[10,13]$. After a series of observations, it has been reported that $\mathrm{H}_{2} \mathrm{O}_{2}$ induction of $\mathrm{SAR}$ genes requires $\mathrm{SA}$ in NahG Arabidopsis plants. [14]. The high levels of $\mathrm{H}_{2} \mathrm{O}_{2}$ also found to stimulate biosynthesis of salicylic acid. [14, 15, 16]. Among the three forms of catalases: Class I catalases are expressed in photosynthetic tissues, Class II catalases are expressed in vascular tissues and Class 3 catalases are expressed in seeds and reproductive tissues. [17]. These reports strongly 
suggest that $\mathrm{H}_{2} \mathrm{O}_{2}$ does not function in the downstream of SA regulating the $\mathrm{PR}$ genes through inhibition of catalase.

In this work, we have developed a model to show the inhibition of catalase by SA through computational approach. We have been working on geminiviral infections in Cucurbitaceae family [18], we have studied the mechanism of salicylic acid in Cucurbita pepo (pumpkin). The homology model of catalase of Cucurbita pepo was modeled and different binding modes of SA with catalase has been demonstrated using Autodock tools.

\section{MATERIALS AND METHODS 2.1. Retrieval of Protein Sequences and Templates of Catalase.}

The protein sequence of the catalase gene of Cucurbito pepo was retrieved from NCBI (National Centre for Biotechnology Information). The protein sequence (Accession number: BAA09506.1) was chosen and downloaded from the website. The retrieved sequence was then submitted in BLASTp (http://www.ncbi.nlm.nih.gov/BLAST) and homology search was performed against the Protein Data Bank (PDB) (http://www.rcsb.org/). The following criteria were followed to determine the protein sequence of Cucurbito pepo: total score, query coverage $(\%)$, expected value, and $\%$ identity.

\subsection{Physico-Chemical Property Analysis of Catalase Protein of CUCURBITA Pepo}

The physico-chemical property of the catalase protein was determined using Protparam tool (http://web.expasy.org/protparam/) [19]. Protparam computes molecular weight, Theoretical pI, amino acid composition, total number of positively and negatively charged molecules, extinction coefficients, the half-life, Instability index, Aliphatic Index and Grand Average of hydropathicity (GRAVY).

\subsection{Homology Modeling of Catalase Protein.}

The modeling of Cucurbita pepo protein structure was done based on homology comparative modeling strategy. For achieving this purpose, Modelerv9.10 [20] was used. The software aligned the query sequence with the known template structures. This further calculates a model containing all nonhydrogen atoms. The spatial restraints were automatically satisfied and the protein structures were loop-modelled and the models can be optimized. The optimized model was used for further studies. The energy of the modeled structure was minimized using MolSoft ICMPro [21].

\subsection{Evaluation of the Structure of Modeled Catalase.}

The predicted protein model was subjected to evaluation of its quality. The evaluation of protein structure was carried out in SAVES (http://nihserver.mbi.ucla.edu/SAVES/) server. SAVES comprises of a package of Procheck, What_check, Verify_3D, Errat and Prove. The PROCHECK [22] calculates the Ramachandran plot to check the quality of protein structure based on the residence of residues in the favoured region, allowed and disallowed region. With the increase in the number of residues in the favoured regions, the quality of the protein structure increases. The threshold percentage for the Ramachandran plot is $85 \%$.

\subsection{Prediction of Binding Pocket and the Flexible Residues in Catalase Model.}

The possible binding pockets of the salicylic acid on catalase were predicted using CASTp server [23]. The modeled protein structure was submitted in the server for analysis. The binding pockets were analyzed based on the area and volume of the pocket. All the atoms of the amino acid residues were selected for further studies. Totally 4 pockets were chosen for study. Moreover, the flexible residues that cooperate with the ligand were predicted using FLEXpred server [33]. The server segregates the residues based upon their flexibility and rigidity. The protein sequence was submitted in the server and the parameters were computed.

\subsection{Docking of Catalase with Salicylic Acid.}

Docking simulations were performed using AutoDock version 4.2 for predicting the protein-ligand interactions based on Madeswaran et al [40]. AutoDock using Lamarckian Genetic Algorithm (LGA) performs automated docking with the flexible residues at the active site of catalase. The hydrogen atoms were added to the protein and the non-polar hydrogen atoms were merged. Kollman charges are calculated for each atom of the macromolecule in AutoDock version 4.2. Torsions were applied on the ligand molecule. The flexible residues SER164, SER246, SER437, SER112 of pockets 1 to 4 were chosen for study of the enzyme catalase, respectively. AutoDock requires pre-calculated grid maps, one for each atom type present in the flexible molecules being docked and it stores the potential energy arising from the interaction with rigid macromolecules. This grid must surround the region of interest in the rigid molecule. The grid box size was set at $60 \times 60 \times 60 \AA$ (x:y:z) in dimension was centered on the geometry center of the target protein and were calculated for each of the following atom types: HD, C, A, N, OA, and SA, corresponding to all possible atom types in the protein (Table 1).

Table 1 : Pockets predicted using CASTp server.

\begin{tabular}{|r|l|l|l|l|}
\hline \multicolumn{1}{|c|}{ S.No } & $\begin{array}{l}\text { Pock } \\
\text { ID }\end{array}$ & Area & Volume & Amino acids \\
\hline 1. & 63 & 4040.7 & 7510.4 & PERVHCSIGFKNDLYTQ \\
\hline 2. & 62 & 1751.8 & 4409.5 & MDYRHPSFNAWTVKEI \\
\hline 3. & 61 & 709.7 & 1339.6 & NFPIDGSVKHRVQTESW \\
\hline 4. & 60 & 352.1 & 359 & IHTLRDPFQNEA \\
\hline
\end{tabular}

Atomic affinity potentials were pre-calculated to evaluate the energy for each atom in the ligand molecule. During the AutoGrid procedure, the target enzyme was implanted on a three dimensional grid point [40]. The energy of interaction of each atom in the ligand was encountered. We have selected important docking parameters for the LGA as follows: population size of 150 individuals, 2.5 million energy evaluations, maximum of 27,000 generations, number of top individuals to automatically survive to next generation of 1 , mutation rate of 0.02 , crossover rate of $0.8,10$ docking runs, and random initial positions and conformations. The probability of performing local search on an individual in the population was set to 0.06 . AutoDock was run several times to get various docked conformations, and used to analyze the predicted docking energy. The binding sites for these molecules were selected based on the ligand-binding pocket of the templates [42]. For each ligand, ten best poses were generated and scored using AutoDock 4.2 scoring functions [40].

\section{RESULTS}

\subsection{Physico-Chemical Characterization of Catalase.}

The protein sequence was retrieved from NCBI since the structure of catalase was not available in PDB (Fig. 1)

The accession number of the retrieved protein sequence is P48350: Catalase isoenzyme-1 of Cucurbita pepo which has 492 amino acids. The predicted molecular weight of the protein 
$>$ gi $\mid 862452$ | dbj|BAA09506.1| catalase [Cucurbita pepo] KGEFEVTHDITUSCADELRAPGVODVIVRESTVIHTRGSDETLRDPRGEAVKFY PVFF IRDGMKETRHVHPLKPNPKSHIOENWRILDFFSHHPESLNMFSFLFDD IGIPODYRHMD GSGVNTY TL INKAGKAHYVKFHWRPTCGVKSLLEEDAIRVGGSNHSHATODLYDS IAAGNYPEWKLF IOT IDPDHED KYDFDPLDVTKTWPED ILPLQPVGRMVLNKNIDNFFAENEQLAFCPAIIVPGVYYSDDKLLQTRIFSYAD TQRHRLGPNYLQLPANAPKCAHHNNHHEGFMNFMHRDEEVNYFPSRFDPSRHAERYPHPPAVCSGKRER IIEKENNFKEPGERYRSWTPDRQERFVRRWVALSDTRVTHEIRS IWISYWSQADRSLGQKLASHLNVI

1: Peptide sequence of catalase enzyme from pepo accession number BAA09506.

Cucurbita

is 57070.1 . The theoretical $\mathrm{pI}$ of the catalase protein is 6.96 . This suggests that the net charge is zero at solutions with $\mathrm{pH}$ 7.0. This is because the number of negatively and positively charged residues is 61 and 59, respectively. Since the numbers of the positive and negative residues are nearly equal, the charge of the catalase enzyme is almost neutral. The extinction coefficient of catalase enzyme measured at $280 \mathrm{~nm}$ in water is $80705 \mathrm{M}^{-1} \mathrm{~cm}^{-1}$. The estimated half-life of catalase is 30 hours which corresponds to the mammalian reticulocytes. The instability index (II) is computed to be 39.75 . The threshold value of the instability index is 40 below which the protein is stable and above which the protein is instable in the test tube. The value of instability index is less than 40 and found to be stable. The aliphatic index insists that the volume of the protein occupied by the aliphatic side chains (alanine, valine, isoleucine and leucine). The aliphatic index of catalase protein is 67.97. The grand average of hydropathicity (GRAVY) is -0.640 . Since the GRAVY is negative the protein is found to be hydrophilic. Therefore, it is revealed that the protein is stable, hydrophilic in nature which has extinction coefficient of $30 \mathrm{hrs}$ [19].

\subsection{Secondary Structure Prediction}

The localization of secondary structure elements were predicted using Geneious Pro v 5.1.7. The predicted model of catalase protein consists of 20 alpha helices, 29 beta strand, 50 coils and 57 turns. Predict Secondary Structure uses original Garnier Osguthorpe Robson algorithm (GOR -I) for predicting protein secondary structure (Fig. 2).

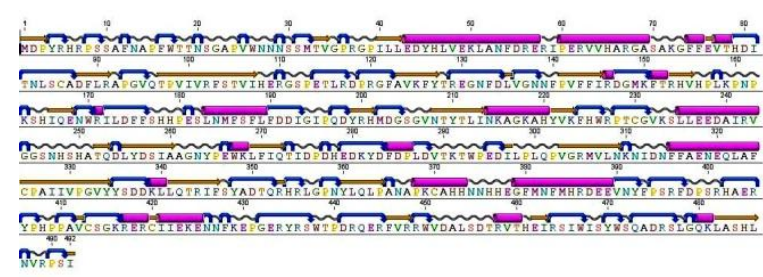

Fig 2. Secondary structure predicted from the peptide sequence of catalase enzyme using Garnier Osguthorpe Robson algorithm.

\subsection{Homology Modeling of Catalase}

The three dimensional model of a protein structure reveals incredible insights into the structural relationship with its function. Homology or comparative modeling is the most common structure prediction method. Upon a PSI-BLAST search against the Protein Data Bank (PDB), 1DGG_A, 1DGH_A 1QQW_A, 3NWL_A was identified as the best templates available for the homology modeling of the catalase with $51 \%$, $44 \%, 41 \%, 41 \%$ sequence identity, respectively to over $96 \%$ query coverage. The query sequence and template structures were then provided as inputs in Modeller9v10 [20] to generate the 3D model of catalase. The DOPE score of the modeled protein is 50806.36 (Fig. 3).

\subsection{Energy Minimization, Quality Assessment and Visualization}

The model generated using Modeller9v10 was subjected to energy minimization and assessed for both geometry and energy aspects using Molsoft ICM-Pro [21].

The quality factor of the final modeled protein is $87.336 \%$. Several structural assessment methods like Ramachandran Plot and RMSD were carried out on the predicted model of catalase to check the reliability and the quality of the protein structure. Ramachandran plot was constructed in PROCHECK [22] in the Structure Analysis and Verification Server (SAVES).

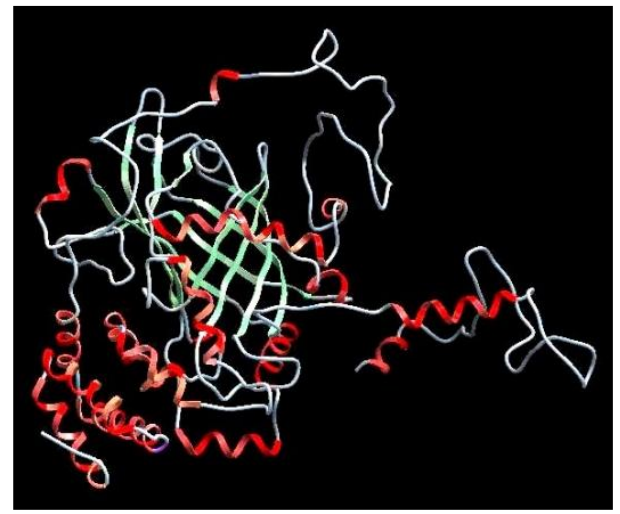

Fig 3. Three dimensional structure of catalase-1 enzyme predicted using Modeller 9v10

The results reveal that only one (Val207) of the total 492 residues were present in the disallowed region whereas another 2 residues were localized in the generously allowed region (Fig. 4).

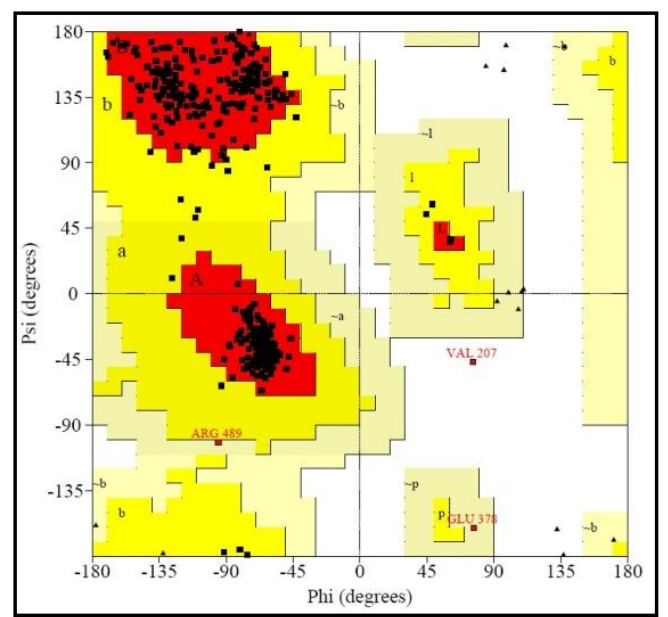

Fig 4. Ramachandran Plot obtained from PROCHECK module of the SAVES server.

There are 404 residues i.e. $94.6 \%$ of amino acid residues were found to reside in the most favoured regions. The usual nature of stereo-chemical property is best measured by G-factor for dihedral angles. The G-factor values below -0.5 represent unusual property while those values below -1.0 are highly unsual. The overall G-factor for dihedral is -0.12 which represents the protein is unusual. In summary, Ramachandran plot and G-factor values denotes that the backbone dihedral angles, PHI and PSI, in the three-dimensional model of catalase protein are well within acceptable limits.

The root mean square deviation (RMSD) indicates the degree at which the template structure and the modeled protein are similar: the lower RMSD denotes higher similarity between structures. The template structure as well as the modeled structure was superimposed to calculate the RMSD between them using 
Molsoft ICMPro. The RMSD values are $0.60 \AA$, $0.60 \AA, 0.69 \AA$ and $0.67 \AA$ with the protein templates 2J2M_A, 1M7S_A, 7CAT_A and 1DGH_A respectively (Fig. 5).

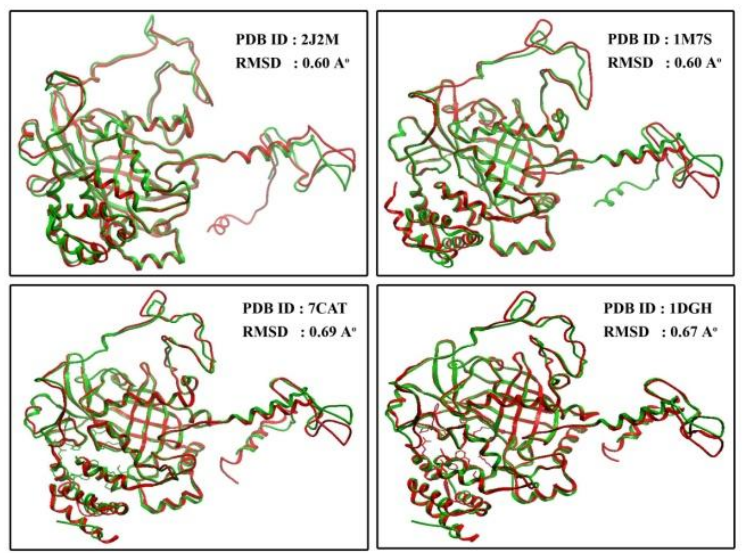

Fig 5. Superimposed structures of modeled catalase enzyme

Collectively, quality factor, Ramachandran plot characteristics and the RMSD values represent that the modeled protein possesses good quality and can be further reproduced for docking studies.

\subsection{Geometry of the Active Site}

The CASTp server used Weighted Delauney Triangulation and the alpha complex for shape measurements. The surface accessible pockets as well as interior inaccessible cavities, for proteins and other molecules were identified and measured. It measures the area and volume of each pocket and cavity, both in solvent accessible surface (SA, Richards' surface) and molecular surface (MS, Connolly's surface) [23, 24]. The prediction of binding site in a protein is a fundamental step in molecular docking. In fact, predicting an apt pocket on the surface of the target protein will be useful for finding out a suitable drug. CASTp server was used because the server uses the alpha shape and the pocket algorithm develo The quality factor of the final modeled protein is $87.336 \%$. Several structural assessment methods like Ramachandran Plot and RMSD were carried out on the predicted model of catalase to check the reliability and the quality of the protein structure. Ramachandran plot was constructed in PROCHECK [22] in the Structure Analysis and Verification Server (SAVES). ped in computational geometry [25] to delineate and measure the surface pockets and voids in proteins. Four different pockets were chosen for study: Pocket 63 , $62,61 \& 60$. The information on area and volume of the binding pocket is given in (Table 2; Fig. 6).

Table 2. Grid Box Parameters used in the study

\begin{tabular}{|c|c|c|c|c|c|c|}
\hline \multirow[t]{2}{*}{ Pockets } & \multirow[t]{2}{*}{ Flexible Residue } & \multirow[t]{2}{*}{ Dimension } & \multirow[t]{2}{*}{ Spacing } & \multicolumn{3}{|c|}{ Grid Box Center } \\
\hline & & & & $\begin{array}{l}\mathbf{x} \\
\text { center }\end{array}$ & y center & $z$ center \\
\hline Pocket 1 & SER164 & $60: 60: 60$ & 0.536 & 19.222 & -21.250 & -6.583 \\
\hline Pocket 2 & SER246 & $60: 60: 60$ & 0.458 & -4.250 & -19.556 & 16.472 \\
\hline Pocket 3 & SER437 & $60: 60: 60$ & 0.564 & 10.694 & 11.139 & 20.972 \\
\hline Pocket 4 & SER112 & $60: 60: 60$ & 0.636 & 2.556 & $\begin{array}{l}-14.778 \\
\end{array}$ & 6.306 \\
\hline
\end{tabular}

\subsection{Energy Minimization of Salicylic Acid}

The 3D chemical structure of salicylic acid was retrieved from PubChem [26]. The energy minimization of salicylic acid was carried out using MM2 and MOPAC module in Chem3D Ultra 2004 at an RMS gradient of $0.0001 \AA$. The three dimensional structure of salicylic acid showed change in the conformation (Fig. 7).

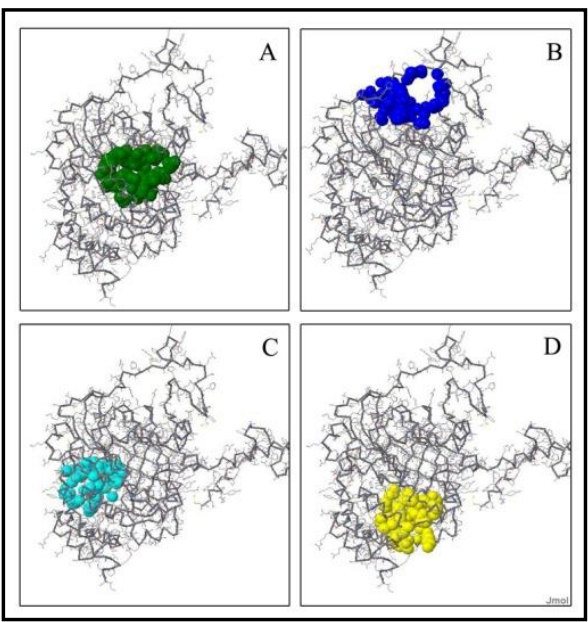

Fig 6. Pocket symmetry of catalase enzyme predicted using CASTp: A) Pocket 63 with area 4040.7 and volume 7510.4; B) Pocket 62 with area 1751.8 and volume 4409.5; C) Pocket 61 with area 709.7 and volume 1339.6; D) Pocket 60 with area 352.1 and volume 359 .

\subsection{Docking of Salicylic Acid to Catalase Enzyme}

The docking experiments performed using AutoDock v4.0 [27] between catalase enzyme and salicylic acid. The scheme of bonding between the enzyme and the inhibitor involves one to three visible hydrogen bonds. The hydrogen binding patterns were compared in each pocket. In pocket 63 , the hydrogen bonds are found between ARG153 and HIS154 (Fig. 7A and 7B). Pocket 62 possess totally three hydrogen bonds each shared with ASN247, HIS248 and SER249 which maintains its stability (Fig. 7C and 7D). In pocket 61 , the stability between the salicylic acid and the enzyme is brought about by only one hydrogen bond formed with the amino acid residue LYS429 (Fig. 7E and 7F) whereas in pocket 60 , the hydrogen bonds are formed between HIS65 and ASN138 (Fig. 7G and 7H). The hydrogen bonds bring about the stability or adjust the protein conformation to make them more stable. 


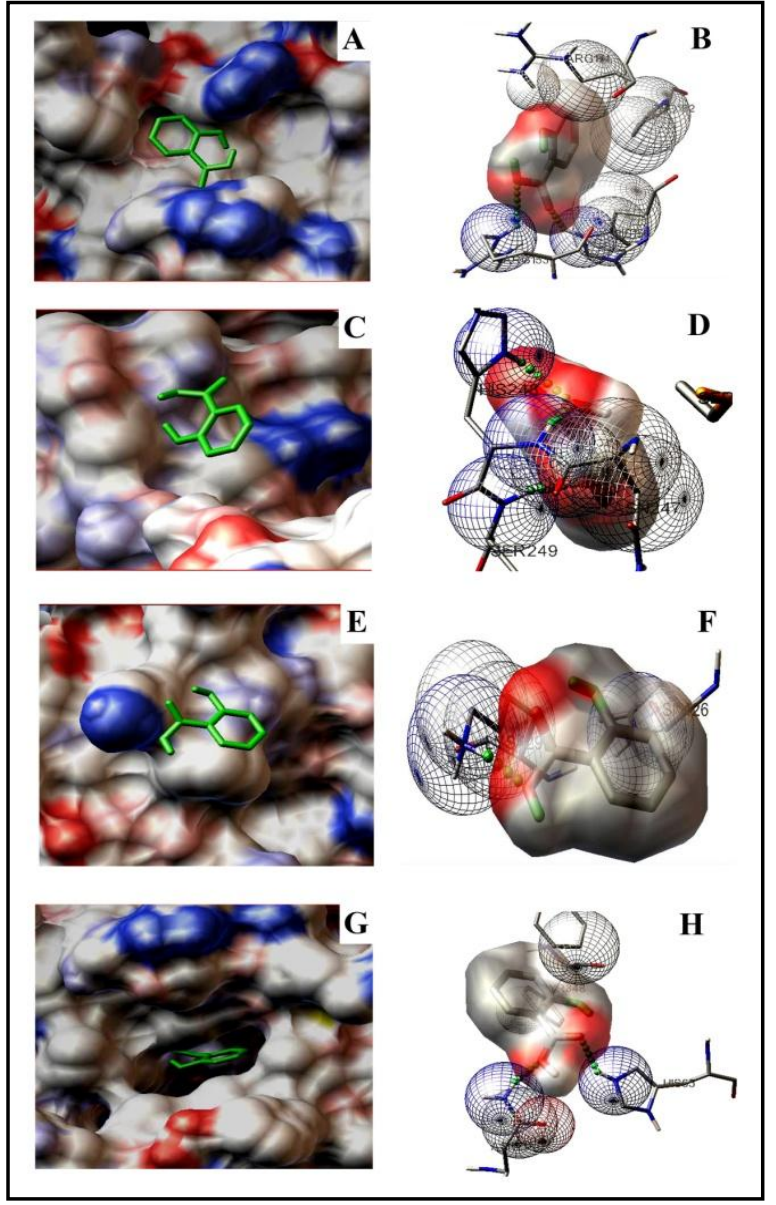

Fig 8. Docking of salicylic acid in different pockets of modeled catalase enzyme. A) salicylic acid bound to pocket 63, B) Hydrogen bonding pattern of salicylic acid with catalase in pocket $63 \mathrm{C}$ ) salicylic acid bound to pocket 62, D) its hydrogen bonding pattern with catalase enzyme in pocket $62, E$ ) salicylic acid bound to the pocket $61, F)$ bonding pattern of salicylic acid in pocket $61, \mathrm{G}$ ) salicylic acid bound to the pocket $60, \mathrm{H}$ ) its hydrogen bonding pattern with the pocket 60 of catalase.

\section{DISCUSSION}

Catalase being the common tetrameric heme containing enzyme persists in all living organisms that are exposed to oxygen, functions to catalyze the decomposition of hydrogen peroxide to water and oxygen $[28,29]$. The enzyme catalase is the most extensively studied class of enzymes.

$$
2 \mathrm{H}_{2} \mathrm{O}_{2} \rightarrow 2 \mathrm{H}_{2} \mathrm{O}+\mathrm{O}_{2}-\cdots \text { [ - [30] }
$$

The regular physiological role of catalase is to remove $\mathrm{H}_{2} \mathrm{O}_{2}$, a reactive oxygen species (ROS) and preventing the cell damage. Among the three isoforms of catalases, CAT-1, CAT-2, CAT-3; CAT-1 is known to highly express during treatments including cold and abscissic acid. In general, CAT-1 is expressed under stress condition. In certain circumstances, bacterial infection induced CAT-2 expressions in potato [31].

Salicylic acid is a potent inducer of plant defence responses including synthesis of pathogen-related protein (PR proteins). The SA-binding protein has been known to be catalase, on inhibition, reactive oxygen species gets accumulated in the cell As result, accumulated ROS induces the production of PR-1 proteins. These PR-1 proteins act against the pathogen for instance, tobacco mosaic virus and prevents its spreading.

The intracellular accumulation of $\mathrm{H}_{2} \mathrm{O}_{2}$ destroys the cells by forming necrotic lesions and further induces programmed cell death. In our study, we have investigated the binding pattern of salicylic acid in 4 different pockets (ID: 63,62,61,60). The structure of catalase enzyme is unavailable in the repository. Therefore, the sequence has been retrieved from NCBI and the homology model was constructed using Modeller9v10. According to Ramachandran plot, $94.6 \%$ of the amino acid residues were localized in the most favoured regions. It is only a minimum of $0.2 \%$ of the amino acid residues reside in the disallowed region. This shows that the model possesses high quality which is due to the greater query coverage and sequence identity between the protein templates. This can be further proved by the low RMSD values between the superimposed structures [32]. The lower RMSD value $0.60 \AA$ between the templates $2 J 2 \mathrm{M}$ and $1 \mathrm{M} 7 \mathrm{~S}$ reveals the closeness and the quality of the structure. The other two templates 7CAT and 1DGH did not show much difference (Fig. 5). CASTp predicted many binding pockets in the modeled protein, among which only 4 pockets $(63,62,61,60)$ were chosen for study based on the area and the volume of the binding pocket (Fig. 6). Further, FlexPred calculated serine in all the four pockets viz, SER164, SER246, SER437 and SER112 as flexible residues in pockets $63,62,61,60$ respectively. FlexPred calculates the flexible residues based on the sequence derived information and solvent accessibility of residue position [33].

Salicylic acid has bound with the receptor binding pocket through inter-atomic and inter-molecular forces between the ligand and the protein complexes [34]. The intermolecular energies in the pockets 63 and 60 are found to be lesser than in other pockets (Table 3). According to Morris et al [27], a generation consists of five stages: mapping and fitness evaluation, selection, crossover, mutation, and elitist selection, in that order. Mapping translates from each individual's genotype to its corresponding phenotype, and occurs over the entire population. This allows each individual's fitness to be evaluated. This is the sum of the intermolecular interaction energy between the ligand and the protein, and the intramolecular interaction energy of the ligand. Free energy (Gibbs, $\Delta \mathrm{G}$ ) is represented by a master equation, broken down into six terms to model dispersion/repulsion, hydrogen bonding, electrostatic interactions, deviation from covalent geometry, internal ligand torsional constraints, and desolvation effects [27].

$$
\Delta \mathrm{G}=\Delta \mathrm{G}_{v d w}+\Delta \mathrm{G}_{\text {hbond }}+\Delta \mathrm{G}_{\text {elec }}+\Delta \mathrm{G}_{\text {conform }}+\Delta \mathrm{G}_{\text {tor }}+\Delta \mathrm{G}_{\text {sol }}
$$

The energies were calculated by placing the protein inside a userdefined three dimensional grid and various probes were allowed to systematically encounter every grid point. Each probe consists of an atom type found in the ligand whose dispersion/repulsion energies and, if appropriate, hydrogen-bonding energies are calculated for all receptor atoms within $8 \AA$ of each grid point [27].

The measure of the effectiveness of a ligand to inhibit the biological and biochemical function is represented as half maximal inhibitory concentration (IC50). The relationship between the inhibition constant $(\mathrm{Ki})$ to concentration of inhibitor which inhibits 50 percent of the enzyme (I50) is dependent on the type of inhibition and the mechanism of the reaction. I50 will be equal to $\mathrm{Ki}$, if the non-competitive or an uncompetitive inhibitor is studied in a monosubstrate enzymatic reaction [35]. According to our work, monosubstrate, i.e. only one ligand has been studied against catalase enzyme. The pockets 63 and 60 possess inhibition constants of $414.16 \mu \mathrm{M}$ and $525.23 \mu \mathrm{M}$, respectively, at which the ligand has the potential to produce half maximum inhibition at that concentration. The ligand has shown to inhibit at micromolar concentration in other 2 pockets and their binding energies are also relatively higher (Table 3 ). Therefore, lower the $\mathrm{kI}$, lower the concentration of inhibitor required to lower the rate of enzyme activity. 
Table 3 : The binding energy and inhibition constant parameters

\begin{tabular}{|c|c|c|c|c|c|c|c|c|}
\hline S.No & Pocket ID & $\begin{array}{c}\text { Binding } \\
\text { Energy }\end{array}$ & $\begin{array}{c}\text { Inhibition } \\
\text { Constant }\end{array}$ & $\begin{array}{c}\text { Intramolecular } \\
\text { energy }\end{array}$ & $\begin{array}{c}\text { Internal } \\
\text { Energy }\end{array}$ & $\begin{array}{c}\text { Torsional } \\
\text { Energy }\end{array}$ & $\begin{array}{c}\text { Unbound } \\
\text { Extended } \\
\text { Energy }\end{array}$ & $\begin{array}{c}\text { Reference } \\
\text { RMS }\end{array}$ \\
\hline 1. & Pocket 63 & -4.61 & $414.16 \mu \mathrm{M}$ & -5.45 & -0.45 & 0.82 & -0.45 & 77.79 \\
\hline 2. & Pocket 62 & -4.01 & $1.14 \mathrm{mM}$ & -4.36 & -0.76 & 0.82 & -0.28 & 76.95 \\
\hline 3. & Pocket 61 & -3.57 & $2.42 \mathrm{mM}$ & -4.28 & -0.32 & 0.82 & -0.21 & 106.32 \\
\hline 4. & Pocket 60 & -4.47 & $525.23 \mu \mathrm{M}$ & -5.14 & -0.66 & 0.82 & -0.51 & 77.83 \\
\hline
\end{tabular}

In fact, lower the binding energy, stronger the binding of ligand to the protein. The binding energy denotes the binding affinity of protein to the ligand. The binding affinity represents the potency of the complex. Both the enthalpy and entropy contributes to binding energy in most of the biological systems [36]. In general, potency refers to the reciprocity of both the binding affinity and ligand efficacy. The intermolecular forces play an important role in defining the binding affinity of a ligand molecule to the receptor. As mentioned previously, lower the binding energy of the complex proves the potency of ligand in the pocket. From our results, the structure has been segregated in to three categories in terms of energy, a) higher potency $(<-8.56)$ b) moderate potency $(-8.00$ to $-7.00 \mathrm{kcal} / \mathrm{mol})$. The binding of salicylic acid is efficient in the pockets 63 and 60 as their binding energies are much lower i.e. $-4.61 \mathrm{kcal} / \mathrm{mol}$ and $-4.47 \mathrm{kcal} / \mathrm{mol}$, respectively. The electronic make-up of the functional groups predicts the binding conformation of ligand to the protein structure. The active site enhances the enzyme to bind to its substrate and catalyze through many different weak interactions because of its non-polar microenvironment [37].

The weak interactions include van der Waals forces, hydrogen bonding and electrostatic interactions [38]. The free energy of binding is calculated using

$$
\Delta \mathrm{G}=\Delta \mathrm{H}-\mathrm{T} \Delta \mathrm{S} \text { (at constant temperature) } \rightarrow[36]
$$

where $\Delta \mathrm{G}$ represents the Gibb's Free Energy of Binding, $\Delta \mathrm{H}$ denotes the enthalpy of the reaction, $\mathrm{T}$ is the temperature at which the reaction takes place and $\Delta S$ denotes the change in the entropy of the reaction between the enzyme and the ligand [39]. The moderate binding energies were observed in pockets 62 and 61 were -4.01 and $-3.57 \mathrm{kcal} / \mathrm{mol}$, respectively. This suggests that these molecules have lower binding affinity than high potent drugs (Table 3). The conformation is a combination of translation, quaternion and torsion angles and has been characterized by intermolecular energy, internal energy and torsional energy. The total binding energy of the protein-ligand complex has been estimated using the following equation.

Binding Free Energy = intermolecular energy + internal energy + torsional free energy - unbound extended energy.

Salicylic acid molecule forms hydrogen bond with ARG153 and HIS154 in Pocket 63 (Fig. 7B shown as green spheres). The $-\mathrm{OH}$ group of the ligand participate in the hydrogen bonding with the $\mathrm{NH}$ group of amino acids in the pocket 63 of catalase enzyme. In pocket 60 , the $-\mathrm{OH}$ group of salicylic acid molecule forms hydrogen bonding with the $-\mathrm{NH}$ group of HIS248 and SER249 residues (Fig. $7 \mathrm{H}$ ) whereas the $-\mathrm{OH}$ group of salicylic acid molecule in the pocket 62 has formed hydrogen bonding with the -NH group of HIS248 and SER249 (Fig. 7D).

But in pocket 61 the $-\mathrm{OH}$ group of salicylic acid formed hydrogen bonding with -NH group of LYS429 residue (Fig. 7F).

Fig. 7 represents the conformation of protein-ligand complexes.
From these results, it is inferred that the binding energy is inversely proportional to the binding affinity of the ligand. Hence, with the decrease in the binding energy of the receptorligand complexes, the efficiency of the ligand to the enzyme increases. In this case, salicylic acid has greater affinity to bind in the pocket 63 but less likely to bind in the pocket 62,61 and 60 [43].

\section{ACKNOWLEDGMENTS.}

This work was financially supported by University Grants Commission (UGC), Govt. of India, New Delhi.

\section{REFERENCES}

[1] Chester, K.S., (1933). The problem of acquired physiological immunity in plants. Q Rev Biol. 1933; 8: 275324.

[2] Ross, A.F., (1961). Localized acquired resistance to plant virus infection in hypersensitive hosts. Virology 14: 329339.

[3] Ross, A.F., (1961). Systemic acquired resistance induced by localized virus infections in plants. Virology 14: 340-358.

[4] Pil Joon Seo., An-Kyo Lee., Fengning Xiang., Chung-Mo Park., (2008). Molecular and Functional Profiling of Arabidopsis Pathogenesis-Related Genes: Insights into Their Roles in Salt Response of Seed Germination. Plant Cell Physiol. 49: 334-344.

[5] Greenberg, J.T., and Yao N., (2006). The role and regulation of programmed cell death in plant-pathogen interactions. Cell Microbiol. 6: 201-211.

[6] Vanacker, H., Lu, H., Rate, D.N., Greenberg, J.T., A role for salicylic acid and NPR1 in regulating cell growth in Arabidopsis. Plant J. 28: 209-216.

[7] Rate, D.N., and Greenberg, J.T., (2001). The Arabidopsis aberrant growth and death mutant shows resistance to Pseudomonas syringae and reveals a role for NPR1 in suppressing hypersensitive cell death. Plant J. 27: 203-211.

[8] Straus, M.R., Rietz, S., van Themaat, E.V., Bartsch, M., Parker, J.E., (2010). Salicylic acid antagonism of EDS1driven cell death is important for immune and oxidative stress responses in Arabidopsis. Plant J. 62: 628-640.

[9] Jaspers, P., and Kangasjärvi, J., (2010). Reactive oxygen species in abiotic stress signaling. Physiol. Plantarum. 138: 405-413.

[10] Chen Z, Silva H, Klessig DF. Active oxygen species in the induction of plant systemic acquired resistance by salicylic acid. Science 262 (1993) 1883-1886.

[11] Durner J, Klessig DF. Inhibition of ascorbate peroxidase by salicylic acid and 2,6-dichloroisonicotinic acid, two inducers 
of plant defense responses, Proc Natl Acad Sci USA 1995; 92: 11312-11316.

[12] Rivas-San Vicente M, Plasencia J. Salicylic acid beyond defence: its role in plant growth and development. J Exp Bot 2011; 62: 3321-3338.

[13] Bi Y-M, Kenton P, Mur L, Darby R, Draper J. Hydrogen peroxide does not function downstream of salicylic acid in the induction of PR protein expression. Plant J 1995; 8: 235 245 .

[14] Neuenschwander, U., Vernooij, B., Friedrich, L., Uknes, S., Kessmann, H., Ryals, J., (1995). Is hydrogen peroxide a second messenger of salicylic acid in systemic acquired resistance. Plant J. 8: 227-233.

[15] Lee, H., Léon, J., Raskin, I., (1995). Biosynthesis and metabolism of salicylic acid. Proc. Natl. Acad. Sci. USA 92: 4076-4079.

[16] Summermatter, C., Sticher, L., Métraux, J-P., (1995) Systemic responses in Arabidopsis thaliana infected and challenged with Pseudomonas syringae. Plant Physiol. 108: 1379-1385.

[17] Amna Mhamdi., Guillaume Queval., Sejir Chaouch., Sandy Vanderauwera., Frank Van Breusegem., Graham Noctor., (2010). Catalase function in plants: a focus on Arabidopsis mutants as stress-mimic models. J. Exp. Bot. 61 (15): 4197 4220 .

[18] Mohammed Riyaz S.U., Deepan, S., Dharanivasan, G., Jesse, M.I., Raja Muthuramalingam., Kathiravan, K., (2013). First report on a variant of Squash leaf curl China virus (SLCCNV) infecting Benincasa hispida in India, New Disease Reports 28: 20.

[19] Gasteiger, E., Hoogland, C., Gattiker, A., Duvaud, S., Wilkins, M.R., Appel, R.D., Bairoch, A., (2005). Protein Identification and Analysis Tools on the ExPASy Server; (In) John M. Walker (ed): The Proteomics Protocols Handbook, Humana Press, pp. 571-607.

[20] Sali, A., Potterton, L., Yuan, F., van Vlijmen, H., Karplus, M., (1995). Evaluation of comparative protein modeling by MODELLER. Proteins 23: 318-326.

[21] Abagyan, R.A., Totrov, M.M., Kuznetsov, D.A., (1994) ICM: A New Method For Protein Modeling and Design: Applications to Docking and Structure Prediction From The Distorted Native Conformation, J. Comp. Chem. 15: 488506.

[22] Laskowski, R.A., MacArthur, M.W., Moss, D.S., Thornton, J.M., (1993). PROCHECK - a program to check the stereochemical quality of protein structures. J. App. Cryst. 26: $283-291$

[23] Joe Dundas., Zheng Ouyang., Jeffery Tseng., Andrew Binkowski., Yaron Turpaz., Jie Liang., (2006). CASTp: computed atlas of surface topography of proteins with structural and topographical mapping of functionally annotated residues. Nucleic Acid Research. 34: 116-118.

[24] Liang, J., Edelsbrunner, H., Woodward, C. (1998). Anatomy of protein pockets and cavities: measurement of binding site geometry and implications for ligand design. Protein Sci. 7: 1884-1897.

[25] Andrew Binkowski, T., Naghibzadeh, S., Liang, J., (2003). CASTp: Computed Atlas of Surface Topography of proteins. Nucleic Acids Res. 31: 3352-3355.
[26] Bolton, E., Wang, Y., Thiessen, P.A., Bryant, S.H., (2008). PubChem: Integrated Platform of Small Molecules and Biological Activities. Chapter 12 IN Annual Reports in Computational Chemistry, Volume 4, Elsevier: Oxford, UK. pp. 217-240.

[27] Morris, G.M., David, S., Goodsell, S., Halliday, R.S., Huey, R., Hart, W.E., Belew, R.K., Olson, A.J., (1998). Automated docking using a Lamarckian genetic algorithm and an empirical binding free energy function. J. Comput. Chem. 19: $1639-1662$

[28] Kirkman, H.N., and Gaetani, G.F., (1984). Catalase: a tetrameric enzyme with four tightly bound molecules of NADPH. Proc. Natl. Acad. Sci. USA. 81: 4343-4347.

[29] Chelikani, P., Fita, I., Loewen, P.C., (2004). Diversity of structures and properties among catalases. Cell Mol. Life Sci. 61: 192-208.

[30] Wojtaszek, P., (1997). Oxidative burst: an early plant response to pathogen infection. Biochem J. 322: $681-692$.

[31] Almeida, J.M., Fidalgo, F., Confraria, A., Santos, A., Pires, H., Santos, I., (2005). Effect of hydrogen peroxide on catalase gene expression, isoform activities and levels in leaves of potato sprayed with homobrassinolide and ultrastructural changes in mesophyll cells. Funct. Plant Biol 32: $707-720$

[32] Vladimir, N.M., and Gordan, M.C., (1994). Significance of Root-Mean-Square Deviation in Comparing Threedimensional Structures of Globular Proteins. J. Mol. Biol 235: 625-634.

[33] Kuznetsov, I.B., and McDuffie, M., (2008). FlexPred: a web-server for predicting residue positions involved in conformational switches in proteins. Bioinformation 3: 134136.

[34] Vincent, T.M., Ernst-Ludwig, F., Hermann, E.G., (1994). Intermolecular forces and energies between ligands and receptors. Science 266: 257 - 259 .

[35] Cheng, Y., and Prusoff, W.H., (1973). Relationship between the inhibition constant (K1) and the concentration of inhibitor which causes 50 per cent inhibition (I50) of an enzymatic reaction. Biochem. Pharmacol. 22: 3099-3108.

[36] Cozzini, P., Fornabaio, M., Marabotti, A., Abraham, D.J., Kellogg, G.E., Mozzarelli, A., (2004). Free Energy of Ligand Binding to Protein: Evaluation of the Contribution of Water Molecules by Computational Methods. Curr. Med. Chem. 11: 1345-1359.

[37] Richard, A.E., Hans, B., Gudrun, S., Andreas, E., Ulrich, B., Wolfram, B., Robert, H., Thomas, P., Rainer, R., Wolfgang, S., (1996). Enzyme flexibility, solvent and 'weak' interactions characterize thrombin-ligand interactions: implications for drug design. Structure 4: 1353-1362.

[38] Baker, E.N., and Hubbard, R.E., (1984). Hydrogen bonding in globular proteins. Prog. Biophys. Mol. Bio. 44: 97-179.

[39] Jencks, W.P., (1981). On the attribution and additivity of binding energies. Proc. Natl. Acad. Sci. USA 78: 40464050 .

[40] Madeswaran, A., Umamaheswari, M., Asokkumar, K., Sivashanmugam, T., Subhadradevi, V., Jagannath, P., (2012). Computational drug discovery of potential TAU protein kinase I inhibitors using in silico docking studies. Orient Pharm. Exp. Med. 12: 157-161. 
[41] Umamaheswari, M., Madeswaran, K., Asokkumar, T., Sivashanmugam, V., Subhadradevi, P., Jagannath, P., (2011). Study of potential xanthine oxidase inhibitors: In silico and in vitro biological activity biological activity. Bangladesh J. Pharmacol. 6: 117-123. .

[42] Madeswaran, A., Umamaheswari, M., Asokkumar, K., Sivashanmugam, T., Subhadradevi, V., Jagannath, P., (2011). In Silico docking studies of lipoxygenase inhibitory activity of commercially available flavonoids. J Comput Method Mol Design 2011; 1: 65-72.

[43] Sakthivelkumar, S., Michael Immanuel Jesse., Veeramani, V., Ramaraj, P., Kathiravan, K., Arumugam, M., Janarthanan, S., (2013). Diversity and analysis of sequences encoded by arcelin genes from Indian wild pulses resistant to bruchids. Process Biochem. 48: 1697-1705. 